\title{
Platelet IP6K1 Regulates Neutrophil Extracellular Trap-Microparticle Complex Formation in Acute Pancreatitis
}

\author{
RAED MADHI ${ }^{1, \#}$, MILLADUR RAHMAN $^{1, \#}$, DLER TAHA ${ }^{1}$, JOHAN LINDERS $^{1}$, MOHAMMED \\ MERZA $^{1,3}$, YONGZHI WANG ${ }^{1}$, MATTHIAS MÖRGELIN ${ }^{2}$ and HENRIK THORLACIUS ${ }^{1, *}$
}

${ }^{1}$ Department of Clinical Sciences, Section of Surgery Malmö, Lund University, 20502 Malmö, Sweden ${ }^{2}$ Colzyx, Medicon Village, 22381 Lund, Sweden

${ }^{3}$ College of Pharmacy, Hawler Medical University, Erbil, Kurdistan Region, Iraq.

One sentence summary: IP6K1 is a novel therapeutic target in acute pancreatitis

Abbreviations: (AP) acute pancreatitis; (ELISA) enzyme-linked immunosorbent assay; (IL-6)

interleukin-6; (i.p.) intraperitoneal; (IP6K1) inositol hexakisphosphate kinase 1; (i.v.) intravenous; (MIP2/CXCL2) macrophage inflammatory protein-2; (MMP-9) matrix metalloproteinase-9; (MP)

microparticle; (MPO) myeloperoxidase; (NETs) neutrophil extracellular traps; (PMA) phorbol myristate acetate; (PBS) phosphate buffered saline; (PNA) platelet-neutrophil aggregates; (PMN)

polymorphonuclear neutrophil; (TGFB1) transforming growth factor beta 1

Conflict of interest statement: The authors have declared that no conflict of interest exists.

${ }^{\#}$ Authors contributed equally to this work

* To whom all correspondence should be addressed:

Henrik Thorlacius, MD, PhD

Department of Clinical Sciences

Section of Surgery, Malmö

Lund University

S-205 02 Malmö, Sweden.

Telephone: +46-40-331000

E-mail: henrik.thorlacius@med.lu.se 


\begin{abstract}
Platelet inositol hexakisphosphate kinase 1 (IP6K1) has been shown to control systemic inflammation. Herein, we examined if platelets and IP6K1 regulate pancreatic tissue injury via formation of NETs in experimental models of acute pancreatitis (AP) in mice. By use of electron microscopy abundant NET formation was observed in the inflamed pancreas. These NETs contained numerous microparticles (MP) expressing CD41 or Mac-1. Platelet depletion reduced deposition of NET-MP complexes in the inflamed pancreas. Circulating platelet-neutrophil aggregates (PNA) were increased and inhibition of P-selectin not only disrupted PNA formation but also reduced NETs formation in the inflamed pancreas. NETs depleted of MPs had lower capacity to provoke amylase secretion and STAT-3 phosphorylation in acinar cells. Taurocholate-induced NETs formation, inflammation and tissue damage in the pancreas were decreased in IP6K1-deficient mice. Thrombin stimulation of mixtures of wild-type platelets and neutrophils resulted in NETs formation but not when IP6K1-deficient platelets were incubated with wild-type neutrophils. Polyphosphate rescue restored thrombin-induced NET formation in mixtures of IP6K1-deficient platelets and wild-type neutrophils. Platelet IP6K1 regulates NET-MP complex formation in the pancreas of mice during induction of AP. Targeting platelet IP6K1 might useful to decrease NET-dependent pancreatic tissue inflammation and tissue injury in patients with AP.
\end{abstract}

KEYWORDS: Chemokines, Histones, Inflammation, Leukocytes, Pancreas 


\section{INTRODUCTION}

Acute pancreatitis (AP) is an unpredictable disease with a wide spectrum of clinical outcome ranging from a mild and reversible condition to a severe and life-threatening disorder characterized by

coagulopathy and respiratory failure (1-3). Management of patients with severe AP is largely restricted to supportive therapies due to limited understanding of the underlying pathophysiology. Convincing data have shown that neutrophil recruitment constitutes a rate-limiting step in tissue damage associated with AP (4-7). Studies have revealed that neutrophil-derived reactive oxygen species (ROS) and enzymes, such as elastase and matrix metalloproteinase-9 (MMP-9) can contribute to tissue damage in AP (8, 9). A recent investigation showed that neutrophil extracellular traps (NETs) play a significant role in trypsin activation, neutrophil recruitment and tissue injury in AP (10). Another study showed that NETs contributes to secretory obstruction in the inflamed pancreas (11). Although NETs appear to be important in the pathophysiology of AP, the mechanisms of NETs formation in AP remain elusive. NETs are decorated with nuclear, cytoplasmatic and granular proteins, which exert important biologic functions (12). Activated neutrophils also shed off microparticles (MPs), i.e. sphere-shaped intact vesicles released from cell membranes with a size less than $1 \mu \mathrm{m}$ (13) and recent evidence has shown that MPs form complexes with NETs via histone-phosphatidylserine interactions (14). These NET-MP aggregates are powerful inducers of thrombin generation via the intrinsic pathway of coagulation (14). However, it is not known whether MPs associates with NETs and contribute to inflammation and tissue injury in AP.

Beside their well-known function in haemostasis and thrombosis, accumulating data suggest that platelets exert pro-inflammatory effects in different models of inflammation (15). For example, several studies have shown that platelets support neutrophil recruitment to sites of inflammation via secretion of CD40L, CCL5 and CXCL4 (16-19). Moreover, convincing data have shown that platelets promote NET formation in response to challenge with bacterial and viral components (20-22) although the role of platelet-mediated NETosis in sterile inflammation remains elusive. Platelets are the main source of inorganic polyphosphates in mammalian cells and can secrete short PolyP polymers (60-100 residues) with potent effects on coagulation (23). Moreover, recent publications indicate that PolyPs can also exert 
pro-inflammatory effects, such as enhancing NF- $\kappa \mathrm{B}$ signaling and vascular permeability as well as activating complement system (24-26). A previous study showed that inositol hexakisphosphate kinase 1 (IP6K1) is a critical enzyme regulating polyphosphate homeostasis in platelets (27). In addition, platelet IP6K1 has been reported to regulate formation of platelet-neutrophil aggregates (PNA) in endotoxininduced lung inflammation, suggesting a pro-inflammatory role in systemic inflammation (28). However, the potential role of IP6K1 in the pathophysiology of AP has never been studied.

Based on the above considerations, we hypothesized herein that platelet IP6K1 might not only be involved in NETs formation but also regulate subsequent organ inflammation and injury in AP. 


\section{RESULTS}

\section{Platelets regulate formation of NET-MP aggregates in AP}

Scanning electron microscopy revealed that challenge with taurocholate triggered formation of extracellular fibrillar and web-like structures in the pancreas compatible with NETs (Figure 1A and C). Moreover, transmission immunoelectron microscopy showed that the neutrophil-derived granule protein elastase as well as histone 4 co-localized with the extracellular DNA in these extracellular fibrillar and web-like structures (Figure 1B), which were not observed in the normal pancreas. An antibody directed against GP1BA was used to deplete mice of platelets (Supplementary Figure 1). We found that platelet depletion markedly decreased NETs formation in the inflamed pancreas (Figure 1A and C). As expected, treatment with DNase I abolished taurocholate-induced NETs formation in the pancreas (Figure 1A and C). In addition, challenge with taurocholate enhanced plasma levels of DNA-histone complexes by 3-fold (Figure 1E). Depletion of platelets reduced DNA-histone complexes in plasma by $86 \%$ in animals with AP (Figure 1E). Taurocholate increased pancreatic levels histone 3 and histone 4 by 3 -fold (Figure 1F) and 4-fold (Figure 1G), respectively. Platelet depletion greatly attenuated histone 3 and histone 4 levels in the inflamed pancreas (Figure 1F and G). Moreover, by applying scanning electron microscopy, we found that taurocholate-induced NETs in the pancreas contained numerous round structures compatible with MPs (Figure 1C). Indeed, transmission electron microscopy revealed that these particles expressed CD41 or Mac-1 (Figure 1D), indicating that these MPs were of platelet and neutrophil origin, respectively. Administration of DNase I abolished NET formation and consequently the density of MP on NETs was close to zero in the pancreas of animals with acute pancreatitis (Figure 1A and C-D). Platelet depletion, which reduced NET formation, also decreased the density of MPs on the remaining NETs (Figure 1C-D) as well as tissue damage (Supplementary Figure 1C-K) and inflammation (Supplementary Figure 2A-F) in the inflamed pancreas. 


\section{Platelet-neutrophil aggregates in AP}

Leukocyte-platelet aggregate formation is a common feature in inflammatory conditions (29-31) and we hypothesized that such complex formation may influence neutrophil activation and NETs formation. It was found that taurocholate challenge markedly enhanced formation of PNA formation in the circulation of mice with AP (Figure 2A-B). Moreover, immunoneutralization of P-selectin markedly reduced taurocholate-induced formation of PNA (Figure 2A-B). Next, we examined if P-selectin-mediated contact between platelets and neutrophils is important for NETs formation. It was found that thrombin stimulation of co-incubated platelets and neutrophils increased formation of DNA-histone complexes (Figure 2I).

Interestingly, inhibition of P-selection abolished thrombin-induced formation of DNA-histone complexes in mixtures of platelets and neutrophils, suggesting that P-selectin-mediated contact between neutrophils and platelets is important for NET formation (Figure 2I). Indeed, immunoneutralization of P-selectin markedly decreased taurocholate-induced NETosis in the inflamed pancreas (Figure 2F-H). Moreover, it was found that inhibition of P-selectin significantly decreased inhibition plasma levels of DNA-histone complexes and pancreatic levels of histone 3 and histone 4 in animals with AP (Figure 2C-E) as well as inflammation (Supplementary Figure 3A-C and K-M) and tissue damage (Supplementary Figure 3D-J) in the pancreas of animals with AP.

\section{NET-MP complexes regulate trypsin activation in acinar cells}

We next asked if MPs attached to NETs play a role in AP. As shown recently (14), PMA stimulation of neutrophils triggers formation of NETs containing numerous MPs (Figure 3A). Co-incubation of neutrophils with calpain and caspase inhibitors resulted in formation of NETs with markedly less MPs (Figure 3B). It was found that NETs depleted of MPs had a significantly lower capacity to trigger amylase secretion from acinar cells in vitro (Figure 3E). Signal transducer and activator of transcription-3 (STAT3) is an important signaling molecule in acinar cells (32). It was found that NETs stimulation markedly increased STAT-3 activity in acinar cells (Figure 3F-G) and that co-incubation with DNase I completely decreased NET-induced phosphorylation of STAT-3 (Figure 3F-G). 
Notably, co-incubation with caspase and calpain inhibitors decreased NET-induced STAT-3

phosphorylation by $44 \%$ (Figure 3F-G). However, co-incubation of acinar cells with neutrophil-derived MPs alone had no effect on amylase secretion (Figure 3E) or STAT-3 phosphorylation (Figure 3F-G). Importantly, co-incubation of neutrophils with calpain and caspase inhibitors had no effect on the levels of PMA-induced DNA-histone complex levels or NETs content of histone 4 (Figure 3C-D). We next examined gene expression of STAT-3-targets IL-6 and TGFB1 in acinar cells. We observed that NET challenge greatly enhanced acinar cell mRNA levels of IL-6 (Figure 3H) and TGFB1 (Figure 3I). Coincubation with DNase I or calpain and caspase inhibitors significantly reduced NET-provoked gene expression of IL-6 and TGFB1 in acinar cells (Figure 3H-I). MPs alone had no effect on mRNA levels of IL-6 and TGFB1 in acinar cells (Figure 3H-I).

\section{IP6K1 regulates NET formation in AP}

By use of electron microscopy, it was found that NETs formation in the pancreas was markedly reduced in IP6K1-deficient mice exposed to taurocholate (Figure 4A-C). Taurocholate challenge increased DNAhistone complexes in plasma by more than 7-fold (Figure 4D). Plasma levels of DNA-histone complexes triggered by taurocholate was decreased by $81 \%$ in animals lacking IP6K1 (Figure 4D). We next quantified tissue levels of histones in the pancreas. Pancreatic levels of histone 3 and histone 4 were low in sham animals (Figure 4E-F). Challenge with taurocholate enhanced pancreatic levels of histone 3 and histone 4 by 52 -fold (Figure 4E) and 25-fold (Figure 4F), respectively. We found that taurocholateprovoked increases in histone 3 and histone 4 levels were reduced by more than $97 \%$ and $87 \%$, respectively, in the inflamed pancreas of IP6K1 gene-deficient mice (Figure 4E-F). Notably, there were no differences in the number of leukocyte subtypes and platelets between wild-type and IP6K1-deficienct animals (Supplementary Figure 5). 


\section{IP6K1-dependent inflammation and tissue damage in AP}

Blood levels of amylase were assessed as an indicator of tissue damage in AP. Challenge with taurocholate enhanced amylase levels by nearly 21-fold (Figure 5A). Taurocholate-induced increase in amylase was decreased by 65\% in IP6K1-deficient animals (Figure 5A). Tissue levels of MPO were used as an indicator of neutrophil infiltration. We found that infusion of taurocholate increased pancreatic MPO activity by 12-fold (Figure 5B). Taurocholate-provoked pancreatic levels of MPO was attenuated by 86\% in mice lacking IP6K1 (Figure 5B). Moreover, taurocholate increased formation of CXCL1 was significantly decreased in IP6K1 gene-deficient animals (Figure 5C). Morphologic examination revealed normal microarchitecture in pancreas tissue from control animals (Figure 5D), whereas taurocholate challenge caused severe destruction of the pancreatic tissue structure characterized by extensive edema, acinar cell necrosis, haemorrhage in the interstitial tissue and massive infiltration of neutrophils (Figure 5E and G-J). IP6K1 knock-out mice were protected from taurocholate-provoked destruction of the tissue architecture (Figure 5F and G-J). For example, taurocholate-induced edema, acinar cell necrosis, haemorrhage and leukocyte accumulation were reduced by $64 \%, 61 \%, 63 \%$ and $62 \%$, respectively, in IP6K1-deficient mice (Figure 5G-J). As part of a systemic inflammatory response in severe AP, activated neutrophils accumulate in the lung and MMP-9 and IL-6 levels increase in the circulation (Figure 5K-M). Herein, we observed that MPO levels in the lung was attenuated by 66\% (Figure 5M), plasma levels of MMP-9 by $80 \%$ (Figure 5L) and IL-6 by 90\% (Figure 5K) in IP6K1 knock-out mice exposed to taurocholate.

\section{The IP6K inhibitor TNP decreases NET formation, inflammation and tissue injury in AP}

Data above suggest that IP6K1 is a potential therapeutic target in AP, and thus, we next wanted to study if TNP, a specific inhibitor of IP6Ks (33), could ameliorate taurocholate-induced inflammation and tissue damage. Again, challenge with taurocholate increased NET formation in the pancreas (Figure 6A).

Treatment with TNP markedly decreased NET formation in the inflamed pancreas (Figure 6A-C). In addition, administration of TNP reduced DNA-histone complex levels in the plasma by $63 \%$ and histone 
3 and 4 levels in the pancreas by $68 \%$ and $67 \%$, respectively, in animals exposed to taurocholate (Figure 6D-F). Notably, we found that administration of TNP attenuated amylase by $48 \%$, pancreatic MPO activity by $75 \%$ and CXCL1 levels by $67 \%$ in mice with AP (Figure 7A-C). Moreover, animals treated with TNP were protected from taurocholate-induced tissue damage (Figure 7I-L). For example, TNP decreased edema by $53 \%$, haemorrhage by $46 \%$ and acinar cell necrosis by $44 \%$ in the inflamed pancreas (Figure 7M-O).

\section{Platelet-derived inorganic polyphosphates regulate NETs formation}

IP6K1 is known to regulate platelet homeostasis of polyphosphate formation (27) and we therefore wanted to examine the role of polyphosphates in formation of NETs. First, we observed that IP6K1 disruption reduced the amount of polyphosphates in platelets (Supplementary Figure 4A-B). Next, we coincubated wild-type or IP6k1-deficient platelets together with wild-type neutrophils. Stimulation of wildtype platelets and neutrophils with thrombin resulted in significantly increased formation of DNA-histone complexes (Supplementary Figure 4C). In contrast, thrombin stimulation of IP6K1-deficient platelets coincubated with wild-type neutrophils led to a 59\% reduction in DNA-histone complexes (Supplementary Figure 4C). Interestingly, polyphosphate rescue dose-dependently restored formation of DNA-histone complexes in thrombin stimulated mixtures of IP6K1-deficient platelets and wild-type neutrophils (Supplementary Figure 4C). By use of confocal microscopy, we observed that thrombin stimulation of wild-type platelets and neutrophils resulted in the expulsion of DNA co-localizing with MPO and citrullinated histone 3, indicating NET formation (Figure 8B). No expulsion of DNA was observed when IP6K1-deficient platelets and wild-type neutrophils were stimulated with thrombin (Figure 8C). Notably, NETs formation was restored when IP6K1-deficient platelets and wild-type neutrophils were rescued by addition of polyphosphate (Figure 8D). In separate experiments, wild type and IP6K1-deficient neutrophils were co-incubated with PMA and it was found that PMA-induced NET formation was independent of IP6K1 in neutrophils (Supplementary Figure 6A-C). 


\section{IP6K regulates NET formation and tissue injury in L-arginine-induced AP}

In order to examine whether IP6K also regulates NET formation, tissue damage and neutrophil recruitment in an alternative experimental model, L-arginine was used to trigger AP. Challenge with Larginine increased NET formation in the pancreas (Supplementary Figure 7A-C), plasma levels of DNAhistone complexes (Supplementary Figure 7D) and pancreatic levels of citrullinated histone 3 formation (Supplementary Figure 7E). Administration of TNP significantly decreased NET formation in animals exposed to L-arginine (Supplementary Figure 7A-E). Moreover, L-arginine challenge increased blood amylase levels as well as pancreatic and plasma markers of inflammation (Supplementary Figure 8A-H). Treatment with TNP reduced levels of blood amylase as well as levels of MPO and pro-inflammatory compounds in the pancreas and plasma (Supplementary Figure 8A-H). Finally, administration of TNP attenuated L-arginine-induced edema, hemorrhage, acinar cell necrosis and leukocyte infiltration in the pancreas (Supplementary Figure 8I-O). 


\section{DISCUSSION}

Due to limited understanding of the underlying pathophysiology and lack of specific treatment, mortality rate of patients with severe AP and systemic complications is still as high as $25 \%$. This study documents that platelets are potent regulators of NET-MP complex formation in the inflamed pancreas. These NETMP complexes activate and stimulate amylase secretion from acinar cells. Moreover, our data show that platelet IP6K1 is a potential target to inhibit NETs formation and tissue damage in AP.

Convincing evidence in the literature suggest NETs play a significant role in the development of AP $(10,11)$. Knowing that platelets facilitate neutrophil activation and recruitment $(16,22,34)$, we hypothesized that platelets might regulate NETs formation in AP. Indeed, we found that platelet depletion markedly reduced deposition of DNA co-localizing with the neutrophil-derived granule protein elastase and histone 4, suggesting that platelets regulates NET formation in the inflamed pancreas. Previous work has shown that platelets contribute to NET formation in response to challenge with bacterial and viral components (20-22) but this study suggest that platelet-mediated NETosis is also in involved in sterile inflammation. Moreover, it was found that taurocholate challenge increased DNA-histone complexes in plasma as well as histone 3 and histone 4 levels in the pancreas. Notably, platelet depletion greatly reduced DNA-histone complexes in plasma and histone levels in the pancreas in animals exposed to taurocholate, which further supports the notion that platelets regulate NETs generation in AP. Knowing that NET-derived histones can directly cause epithelial cell damage (35) and trypsinogen activation in acinar cells (10), our findings indicate that decreased pancreatic levels of histone 3 and histone 4 might help to explain part of the beneficial effect of depleting animals of platelets in AP. A pioneering study in this field showed that TLR4 regulates platelet-mediated NETosis in enodotoxemia (20). Considering that TLR4 has also been reported to play an important role in neutrophil recruitment and tissue damage in $\mathrm{AP}(36)$ it is possible that platelet TLR4 might be involved in NETs formation in the inflamed pancreas, which should be addressed in future studies. NETs are known to be loaded with numerous different nuclear, cytoplasmatic and granular proteins, which contribute to the biological effects of NETs $(12,37)$. We have recently demonstrated that MPs formed during neutrophil activation can bind to NETs and form 
NET-MP complexes, which are important for thrombin generation in sepsis (14). Herein, we observed that taurocholate challenge increased the number of NET-MP aggregates in the pancreas and that depletion of platelets decreased NET-MP aggregates in the inflamed pancreas. Moreover, these NETassociated MPs were found to express Mac-1 or CD41, suggesting that both neutrophil- and plateletderived MPs formed complexes with NETs in the inflamed pancreas. We next asked whether these NETMP aggregates might play an important role in acinar cell biology. By use of electron microscopy, we could confirm that co-incubation of caspase and calpain inhibitors abolished concomitant generation MPs resulting in formation of NETs without MPs. Notably, these MP-depleted NETs were significantly less effective in triggering amylase secretion, STAT-3 phosphorylation and gene expression of IL-6 and TGFB1 in acinar cells compared to NET-MP aggregates. In this context, it should be mentioned that coincubation with caspase and calpain inhibitors did not impact the amount of NET formation or the histone content of NETs depleted of MPs. Thus, these finding suggest that NETs constitute a functional assembly scaffold for MPs in AP.

Several studies have shown that circulating platelet-leukocyte complexes is a frequent phenomenon in a wide range of inflammatory conditions, including reperfusion injury (29), abdominal sepsis (30), pulmonary infections (31), and acute myocardial disease (38). Although, the potential function of such aggregates in inflammation has attracted a lot interest recently the actual role of platelet-leukocyte complexes has not been identified. It was therefore of great interest to examine the role of aggregate formation in platelet-leukocyte interactions in the present study. We found that the percentage of plateletneutrophil complexes increased by 7 -fold in mice with pancreatitis, which is in line with previous observations in AP (34). In addition, it was observed that inhibition of P-selectin not only abolished formation of PNA but also significantly decreased NETs formation in the inflamed pancreas. Moreover, we observed that thrombin stimulation of mixtures of platelets and neutrophil resulted in clear-cut generation of NETs and that immunoneutralization of P-selectin significantly reduced this NETs formation. These findings indicate that physical contact between platelets and neutrophils are important for platelet-mediated NETosis. In this context, it is interesting to note that a recent study reported that 
IP6K1 plays a key role in platelet-mediated neutrophil activation and recruitment in the lung in systematic inflammation via formation of neutrophil-platelet aggregates (28). Thus, we next asked whether IP6K1 could play a role in platelet-dependent NETs generation in AP. We found that taurocholate-induced NETMP complex, histone 3 and histone 4 formation in the pancreas as well as generation of DNA-histone complexes in plasma were markedly reduced in mice lacking IP6K1, showing for the first time in the literature that IP6K1 is a key regulator of NETs formation. It is well accepted that neutrophil infiltration is a rate-limiting step in tissue damage the inflamed pancreas (4-6). Herein, it was observed that the taurocholate enhanced MPO levels and neutrophil extravasation in the pancreas were attenuated by $86 \%$ and 62\%, respectively, in IP6K1 gene-deficient mice. CXC chemokines secreted from resident tissue cells co-ordinate tissue navigation of neutrophils (39). We found that taurocholate caused a clear-cut increase in CXCL1 formation in the pancreas and that generation of CXCL1 was abolished in pancreatitis animals lacking IP6K1, indicating that decreased expression of CXCL1 could help explain diminished neutrophil recruitment and tissue damage in the pancreas of IP6K1-deficient mice exposed to taurocholate. Severe AP is characterized by systemic complications including pulmonary neutrophilia $(6,36)$. Herein, we observed that the taurocholate-induced increase in MPO activity in the lung was greatly decreased in IP6K1 gene-deficient mice. Moreover, IP6K1-deficient animals were protected from increases in systemic levels of IL-6 and MMP-9. In addition, these findings suggest that IP6K1 not only regulates local inflammation but also systemic inflammation in animals with AP. Together, our data show that disruption of IP6K1 not only markedly decreased NETs formation in the inflamed pancreas but also reduced inflammation and pancreatic damage in animals with AP, suggesting that IP6K1 is a key regulator of the development of AP. These findings indicate that IP6K1 could be a useful therapeutic target in AP. This notion is supported by our findings showing that the IP6K specific inhibitor TNP (28, 33), greatly attenuated taurocholate-induced NETs formation, local inflammation and tissue damage in the pancreas as well as systemic inflammation and pulmonary neutrophilia in animals with AP. In addition, we found that TNP also reduced NET formation, inflammation and tissue injury in an alternative model of AP, i.e., L-arginine-induced pancreatitis. 
Having established an important function of IP6K1 in NETs formation and AP, we next wanted to define the role of platelet IP6K1 in mediating NETs formation. We found that thrombin stimulation of mixtures of wild-type platelets and neutrophils triggered clear-cut NET formation. In contrast, thrombin activation of mixtures of IP6K1-deficent platelets and wild-type neutrophils resulted in markedly decreased NETs generation. Moreover, PMA stimulation of IP6K1-deficent neutrophils exhibited intact NET formation. Taken together, these findings suggest that platelet, and not neutrophil, IP6K1 is responsible for NETs formation. In this context, it is interesting to note that a recent study showed that IP6K1 is critical in regulating platelet hemostasis of PolyPs (27). Indeed, we observed herein that IP6K1deficient mice have reduced levels of platelet PolyPs. Accumulating data suggest that PolyPs can exert several pro-inflammatory effects, such as activation of the kallikrein-kinin (26) and complement systems (40). Thus, we hypothesized in the present study that platelet PolyP could be involved in NETs formation. In fact, we found that reduced NET formation in response to thrombin stimulation of IP6K1-deficent platelets and wild-type neutrophils is likely due to decreased production of PolyPs in IP6K1-deficent platelets because this defect was rescued by addition of exogenous PolyP. Thus, these results indicate that platelet IP6K1 is a novel regulator of NETosis via secretion of PolyP, which could have significant impact not only on AP but also other conditions, such as sepsis, infection, and autoimmune diseases, in which NETs play a pathophysiological role. As stated above, PolyPs have pleiotropic effects on inflammation and our present findings do not exclude other mechanisms operating in parallel to plateletmediated NETs formation. For example, it has recently been shown that PolyPs stimulate generation of bradykinin via the intrinsic pathway of coagulation (26). Bradykinin is a potent activator of neutrophils and whether such mechanisms are at work in AP remains to be studied. In this context, it should be mentioned that our findings do not exclude that lack of IP6K1 in vivo could also impact coagulation or other systems independent NETs formation. Nonetheless, considering our present findings together with the fact that PolyPs are a potent stimulus of the coagulation system (26) it could be suggested that blocking IP6K1 could be a useful strategy to concomitantly antagonize pathological inflammation and coagulation constituting key features of patients with severe AP. 
Taken together, this study shows for the first time that platelets regulate formation of NETs in the inflamed pancreas. Moreover, we could document that NETs form aggregates with MPs in the inflamed pancreas and that these NET-MP complexes are potent stimuli for amylase secretion and STAT-3 activation in acinar cells. Our data also demonstrate that IP6K1 is a key regulator of NETs formation, inflammation and tissue damage in AP possibly via PolyP secretion from platelets. Thus, these findings identify a new function of IP6K1 in pancreatitis and suggest that targeting IP6K1 could be beneficial to attenuate local and systemic inflammation in severe AP. 


\section{MATERIALS AND METHODS}

\section{Animals}

Male wild-type and IP6K1 gene-deficient C57BL/6 mice (21-25 g) were housed under standardized conditions at $22^{\circ} \mathrm{C}$ in a 12-hour light dark cycle, fed a laboratory diet with water accessible ad libitum. Mice were anesthetized by intraperitoneal (i.p.) injection of $75 \mathrm{mg}$ of ketamine hydrochloride (HoffmanLa Roche, Basel, Switzerland) and 25 mg of xylazine (Janssen Pharmaceutica, Beerse, Belgium) per kg body weight.

\section{Induction of AP and Experimental Design}

Pancreatitis induction was performed as described previously (43). In brief, anaesthetized animals were laparotomised and a puncture ( $23 \mathrm{G}$ needle) was made through the duodenal wall, opposite to the papilla of vater under microscopic observation. A polyethylene catheter connected to a microinfusion pump (CMA/100, Carnegie Medicine, Stockholm, Sweden) was inserted one mm into the common bile duct. The common hepatic duct was clamped with a clip at the liver hilum to prevent hepatic reflux. Ten $\mu$ l of $5 \%$ sodium taurocholate (Sigma-Aldrich, St. Louis, MO, USA) or $0.9 \%$ sodium chloride was infused into the pancreatic duct at a rate of $2 \mu \mathrm{l} / \mathrm{min}$. Methylene blue was used to enable visual control of pancreatic infusion. The catheter and the bile duct clip were removed and the duodenal puncture was closed before suturing of the abdominal wall. Animals were treated with vehicle, DNase I (Pulmozyme, $5 \mathrm{mg} / \mathrm{kg}$, Roche, Grenzach-Wyhlen, Germany), a control antibody (1.0 mg/kg, rat IgG, Cat. \#C301, Enfret Analytics, Eibelstadt, Germany), an anti-GP1BA antibody (1.0 mg/kg, rat IgG, Cat. \#R300, Enfret Analytics), an anti-P-selectin antibody (2 mg/kg, clone RB40.34, ratIgG, BD Bioscience, San Diego, Calif., USA), or TNP (N2-(m-Trifluorobenzyl), N6-(p-nitrobenzyl) purine (20 mg/kg, Tocris bioscience, Bristol, UK)) prior to taurocholate challenge. Animals were euthanized 24 hours after pancreatitis induction and blood samples were collected from the inferior vena cava for measurement of neutrophilplatelet aggregates, DNA histone complexes, CXCL1, CXCL2, interleukin-6 (IL-6) and MMP-9. Pancreatic tissue was rapidly removed and separated in three for later analyses. One third was used for 
electron microscopy and one third was snap-frozen in liquid nitrogen for biochemical quantification of pancreatic myeloperoxidase (MPO), CXCL1, histone 3 and histone 4 levels. One third was fixed in formalin for histological analysis. Lung tissue was harvested for pulmonary MPO measurement.

\section{L-arginine-induced pancreatitis}

In separate experiments, AP was induced by administration of L-arginine ( $4 \mathrm{~g} / \mathrm{kg} / \mathrm{dose})$ i.p. twice at an interval of $1 \mathrm{~h}$ as described in detail previously (44). Vehicle or TNP (N2-(m-Trifluorobenzyl), N6-(pnitrobenzyl) purine (20 mg/kg, Tocris bioscience, Bristol, UK)) were given i.p. before the first dose of Larginine. Saline-treated animals served as negative controls. All animals were sacrificed $72 \mathrm{~h}$ after induction of pancreatitis.

\section{NETs Generation in Vitro}

Isolated bone marrow neutrophils $\left(4 \times 10^{6}\right.$ cells $\left./ \mathrm{ml}\right)$ were challenged with $50 \mathrm{nM}$ PMA (Sigma-Aldrich, Stockholm, Sweden) for $3 \mathrm{~h}$ at $37^{\circ} \mathrm{C}$ in RPMI 1640. In indicated experiments, cells were co-incubated with caspase (50 $\mu \mathrm{M}, \mathrm{Z}-\mathrm{VAD}-\mathrm{FMK}, \mathrm{R} \& \mathrm{D}$ Systems) and calpain (25 $\mu \mathrm{M}, \mathrm{PD} 150606$, Sigma-Aldrich) inhibitors, which has been shown to generate NETs without MPs (14). Supernatants were discharged and fresh media was added to isolate NETs. Residual neutrophils and NETs were removed through extensive pipetting. The mixture was centrifuged at $200 \mathrm{~g}$ (5 min) to remove cellular components and NETcontaining supernatants were collected. NET-containing supernatants were further centrifuged at $19000 \mathrm{~g}$ (15 min) to collect NETs. For MP preparation, supernatants were subjected to high centrifugation (21 000 $\mathrm{g}$ for $1 \mathrm{~h}$ at $4^{\circ} \mathrm{C}$ ). Supernatants were removed and the pellets finally resuspended in phosphate buffer saline (PBS). Isolated acinar cells were co-incubated with NETs, amylase secretion and STAT-3 phosphorylation and mRNA levels of IL-6 and TGFB1 were determined as described in Supplementary methods.

\section{Statistics}


Graphpad Prism 7 was used for data analysis. Data are presented in box plot (25-75 percentiles) where horizontal line indicates median of the group; whiskers extend from the minimum to the maximum values. Statistical comparisons were performed using non-parametrical tests (Mann-Whitney or ANOVA on ranks followed by Dunnett's multiple comparisons). $\mathrm{P}<.05$ was considered significant and $\mathrm{n}$ represents the number of animals or experiments.

\section{Study approval}

Animal experiments were performed according to the recommendations of the Guide for the Care and Use of Laboratory Animals, published by the US national institutes of health and approved by the ethical committee at Lund University, Sweden (Permit number: 18726/2017). The ARRIVE guidelines (41) and an editorial on the application of ARRIVE guidelines to pharmacological studies (42) were consulted for all in vivo studies involving animals. 
AUTHOR CONTRIBUTIONS: RM, MR, DT, JL, MM and YW performed experiments, analyzed data. RM, MR and JL contributed to the writing. MR and MM2 performed imaging and analysis. HT conceived and design the study and contributed to the writing.

ACKNOWLEDGEMENTS: This work was supported by grants from the Swedish Research Council (2017-01621). Einar och Inga Nilsson Foundation. We are grateful to the staff in the BioEM Lab, Biozentrum, University of Basel, the Core Facility for Integrated Microscopy (CFIM), Panum Institute, University of Copenhagen, and the Microscopy Facility at the Department of Biology, Lund University, for providing highly innovative environments for electron microscopy. We thank Cinzia Tiberi (BioEM lab) and Ola Gustafsson (Microscopy Facility) for skillful work, Carola Alampi (BioEM lab), Mohamed Chami (BioEM lab) and Klaus Qvortrup (FCIM) for practical help with electron microscopy. Raed Madhi is supported by Misan University, College of Science, Iraq. 


\section{REFERENCES}

1. Thandassery RB, Yadav TD, Dutta U, Appasani S, Singh K, and Kochhar R. Dynamic nature of organ failure in severe acute pancreatitis: the impact of persistent and deteriorating organ failure. HPB (Oxford). 2013;15(7):523-8.

2. Salomone T, et al. Coagulative disorders in human acute pancreatitis: role for the Ddimer. Pancreas. 2003;26(2):111-6.

3. Banks PA, et al. Classification of acute pancreatitis--2012: revision of the Atlanta classification and definitions by international consensus. Gut. 2013;62(1):102-11.

4. Sandoval D, et al. The role of neutrophils and platelet-activating factor in mediating experimental pancreatitis. Gastroenterology. 1996;111(4):1081-91.

5. Awla D, et al. Lymphocyte function antigen-1 regulates neutrophil recruitment and tissue damage in acute pancreatitis. Br J Pharmacol. 2011;163(2):413-23.

6. Frossard JL, et al. The role of intercellular adhesion molecule 1 and neutrophils in acute pancreatitis and pancreatitis-associated lung injury. Gastroenterology. 1999;116(3):694701.

7. Abdulla A, Awla D, Thorlacius H, and Regner S. Role of neutrophils in the activation of trypsinogen in severe acute pancreatitis. J Leukoc Biol. 2011;90(5):975-82.

8. Wang HH, Tang AM, Chen L, and Zhou MT. Potential of sivelestat in protection against severe acute pancreatitis-associated lung injury in rats. Exp Lung Res. 2012;38(9$10): 445-52$.

9. Hartman $\mathrm{H}$, et al. P-selectin mediates neutrophil rolling and recruitment in acute pancreatitis. Br J Surg. 2012;99(2):246-55.

10. Merza M, et al. Neutrophil Extracellular Traps Induce Trypsin Activation, Inflammation, and Tissue Damage in Mice With Severe Acute Pancreatitis. Gastroenterology. 2015;149(7):1920-31.e8.

11. Leppkes M, et al. Externalized decondensed neutrophil chromatin occludes pancreatic ducts and drives pancreatitis. Nat Commun. 2016;7:10973.

12. Brinkmann V, et al. Neutrophil extracellular traps kill bacteria. Science. 2004;303(5663):1532-5.

13. Hess C, Sadallah S, Hefti A, Landmann R, and Schifferli JA. Ectosomes released by human neutrophils are specialized functional units. J Immunol. 1999;163(8):4564-73. 
14. Wang Y, et al. Neutrophil extracellular trap-microparticle complexes enhance thrombin generation via the intrinsic pathway of coagulation in mice. Sci Rep. 2018;8(1):4020.

15. Stokes KY, and Granger DN. Platelets: a critical link between inflammation and microvascular dysfunction. J Physiol. 2012;590(5):1023-34.

16. Rahman M, Zhang S, Chew M, Ersson A, Jeppsson B, and Thorlacius H. Platelet-derived CD40L (CD154) mediates neutrophil upregulation of Mac-1 and recruitment in septic lung injury. Annals of surgery. 2009;250(5):783-90.

17. Li G, et al. CD40 ligand promotes Mac-1 expression, leukocyte recruitment, and neointima formation after vascular injury. Am J Pathol. 2008;172(4):1141-52.

18. Hwaiz R, Rahman M, Syk I, Zhang E, and Thorlacius H. Rac1-dependent secretion of platelet-derived CCL5 regulates neutrophil recruitment via activation of alveolar macrophages in septic lung injury. J Leukoc Biol. 2015;97(5):975-84.

19. Wetterholm E, Linders J, Merza M, Regner S, and Thorlacius H. Platelet-derived CXCL4 regulates neutrophil infiltration and tissue damage in severe acute pancreatitis. Transl Res. 2016;176:105-18.

20. Clark SR, et al. Platelet TLR4 activates neutrophil extracellular traps to ensnare bacteria in septic blood. Nat Med. 2007;13(4):463-9.

21. Jenne $\mathrm{CN}$, et al. Neutrophils recruited to sites of infection protect from virus challenge by releasing neutrophil extracellular traps. Cell Host Microbe. 2013;13(2):169-80.

22. Kim SJ, and Jenne CN. Role of platelets in neutrophil extracellular trap (NET) production and tissue injury. Semin Immunol. 2016;28(6):546-54.

23. Kumble KD, and Kornberg A. Inorganic polyphosphate in mammalian cells and tissues. $J$ Biol Chem. 1995;270(11):5818-22.

24. Bae JS, Lee W, and Rezaie AR. Polyphosphate elicits pro-inflammatory responses that are counteracted by activated protein $\mathrm{C}$ in both cellular and animal models. J Thromb Haemost. 2012;10(6):1145-51.

25. Dinarvand $\mathrm{P}$, et al. Polyphosphate amplifies proinflammatory responses of nuclear proteins through interaction with receptor for advanced glycation end products and P2Y1 purinergic receptor. Blood. 2014;123(6):935-45.

26. Muller F, et al. Platelet polyphosphates are proinflammatory and procoagulant mediators in vivo. Cell. 2009;139(6):1143-56. 
27. Ghosh $\mathrm{S}$, et al. Inositol hexakisphosphate kinase 1 maintains hemostasis in mice by regulating platelet polyphosphate levels. Blood. 2013;122(8):1478-86.

28. Hou Q, et al. Inhibition of IP6K1 suppresses neutrophil-mediated pulmonary damage in bacterial pneumonia. Sci Transl Med. 2018;10(435).

29. Kohler D, et al. Phosphorylation of vasodilator-stimulated phosphoprotein (VASP) dampens hepatic ischemia-reperfusion injury. PLoS One. 2011;6(12):e29494.

30. Salat A, et al. Changes of platelet surface antigens in patients suffering from abdominal septic shock. Thromb Res. 1999;95(6):289-94.

31. Schaub RG, Rawlings CA, and Keith JC, Jr. Platelet adhesion and myointimal proliferation in canine pulmonary arteries. Am J Pathol. 1981;104(1):13-22.

32. Zhang $\mathrm{H}$, et al. IL-6 trans-signaling promotes pancreatitis-associated lung injury and lethality. J Clin Invest. 2013;123(3):1019-31.

33. Padmanabhan U, Dollins DE, Fridy PC, York JD, and Downes CP. Characterization of a selective inhibitor of inositol hexakisphosphate kinases: use in defining biological roles and metabolic relationships of inositol pyrophosphates. J Biol Chem. 2009;284(16):10571-82.

34. Awla D, et al. Rho-kinase signalling regulates trypsinogen activation and tissue damage in severe acute pancreatitis. Br J Pharmacol. 2011;162(3):648-58.

35. Saffarzadeh M, et al. Neutrophil extracellular traps directly induce epithelial and endothelial cell death: a predominant role of histones. PLoS One. 2012;7(2):e32366.

36. Awla D, Abdulla A, Regner S, and Thorlacius H. TLR4 but not TLR2 regulates inflammation and tissue damage in acute pancreatitis induced by retrograde infusion of taurocholate. Inflamm Res. 2011;60(12):1093-8.

37. Fuchs TA, et al. Novel cell death program leads to neutrophil extracellular traps. J Cell Biol. 2007;176(2):231-41.

38. Habazettl H, Hanusch P, and Kupatt C. Effects of endothelium/leukocytes/platelet interaction on myocardial ischemia--reperfusion injury. Z Kardiol. 2000;89 Suppl 9:IX/92-5.

39. Bacon KB, and Oppenheim JJ. Chemokines in disease models and pathogenesis. Cytokine Growth Factor Rev. 1998;9(2):167-73. 
40. Wijeyewickrema LC, et al. Polyphosphate is a novel cofactor for regulation of complement by a serpin, C1 inhibitor. Blood. 2016;128(13):1766-76.

41. Kilkenny C, Browne W, Cuthill IC, Emerson M, Altman DG, and Group NCRRGW. Animal research: reporting in vivo experiments: the ARRIVE guidelines. $\mathrm{Br} J$ Pharmacol. 2010;160(7):1577-9.

42. McGrath JC, Drummond GB, McLachlan EM, Kilkenny C, and Wainwright CL. Guidelines for reporting experiments involving animals: the ARRIVE guidelines. $\mathrm{Br} J$ Pharmacol. 2010;160(7):1573-6.

43. Laukkarinen JM, Van Acker GJ, Weiss ER, Steer ML, and Perides G. A mouse model of acute biliary pancreatitis induced by retrograde pancreatic duct infusion of $\mathrm{Na}-$ taurocholate. Gut. 2007;56(11):1590-8.

44. Dawra R, Sharif R, Phillips P, Dudeja V, Dhaulakhandi D, and Saluja AK. Development of a new mouse model of acute pancreatitis induced by administration of L-arginine. $A m$ J Physiol Gastrointest Liver Physiol. 2007;292(4):G1009-18. 

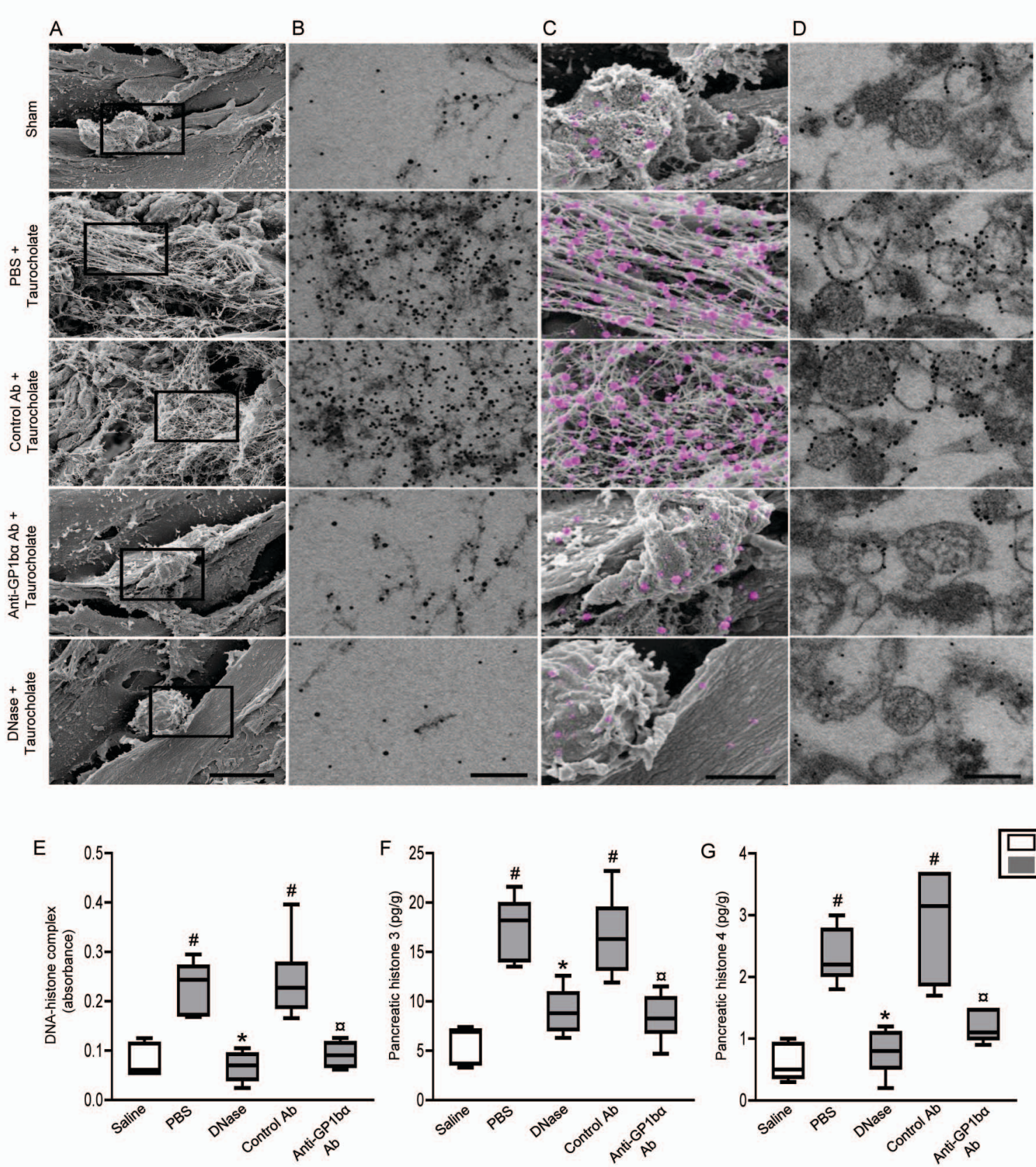

Figure 1. NET-MP complex formation in AP. A) Scanning electron microscopy exhibiting extracellular web-like structures in the inflamed pancreas. Scale bar $=5 \mu \mathrm{m}$. B) Transmission electron microscopy of the indicated area of interest from Figure 1A incubated with gold-labeled anti-histone 4 (large gold particles) and anti-elastase (small gold particles) antibodies. Scale bar $=0.25 \mu \mathrm{m}$. C) A higher magnification of the indicated area of interest from Figure 1A showing fibrillar MPs attached to NETs in the inflamed pancreas. MPs are denoted in pink color. Scale bar $=2 \mu \mathrm{m}$. D) Transmission electron microscopy of the indicated area of interest from Figure 1A incubated with gold-labeled anti-Mac-1 (large gold particles) and anti-CD41 (small gold particles) antibodies. Scale bar $=0.25 \mu \mathrm{m}$. E) Plasma levels of DNA-histone complexes. Pancreatic levels of F) histone 3 and G) histone 4. Pancreatitis (grey boxes) was induced by infusion of sodium taurocholate (5\%) into the pancreatic duct. Sham mice (white boxes) were infused with saline alone. Animals were treated with i.p. injections of the DNase I, a control antibody (Control Ab), an antibody directed against GP1BA (anti-GP1b alpha Ab) or vehicle (PBS) as described in Materials and Methods. Samples were collected 24 hours after induction of pancreatitis. Data represent median (25-75 percentile); whiskers extend from the minimum to the maximum values and $n=5-6 .{ }^{\sharp} P<0.05$ versus sham mice, ${ }^{*} P<0.05$ versus PBS+taurocholate and ${ }^{\text {a }} P<0.05$ versus Control $\mathrm{Ab}+$ taurocholate (Mann-Whitney test). 
A
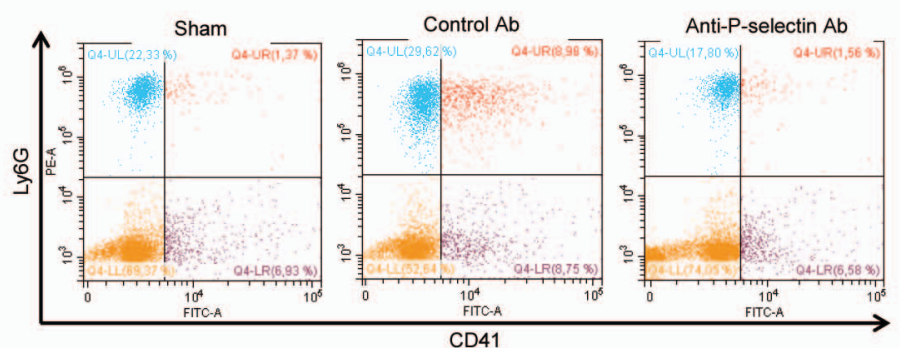

B
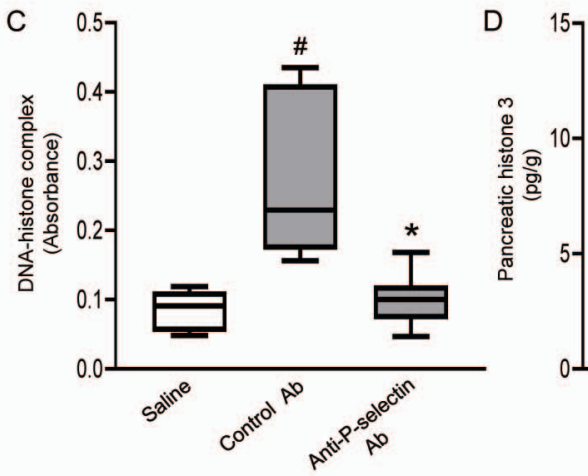

G
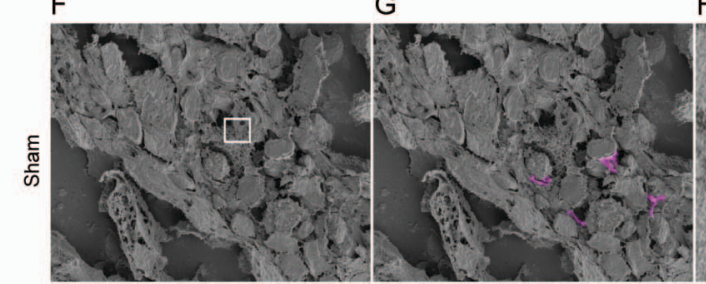

$\mathrm{H}$
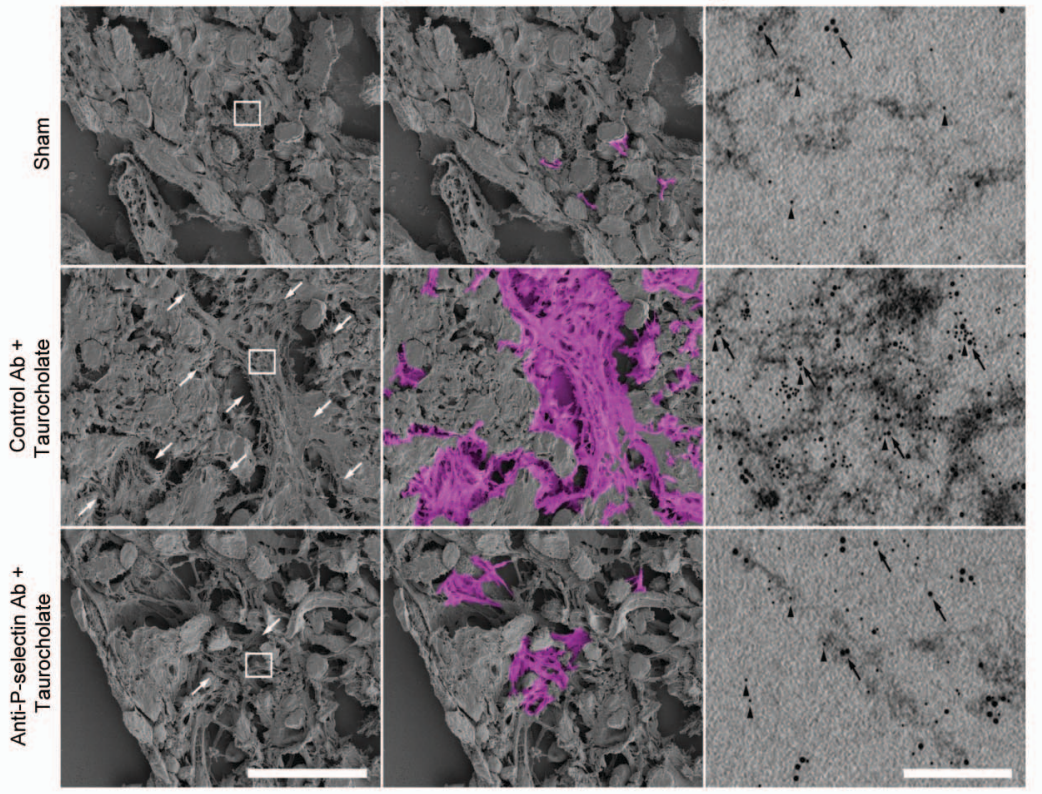

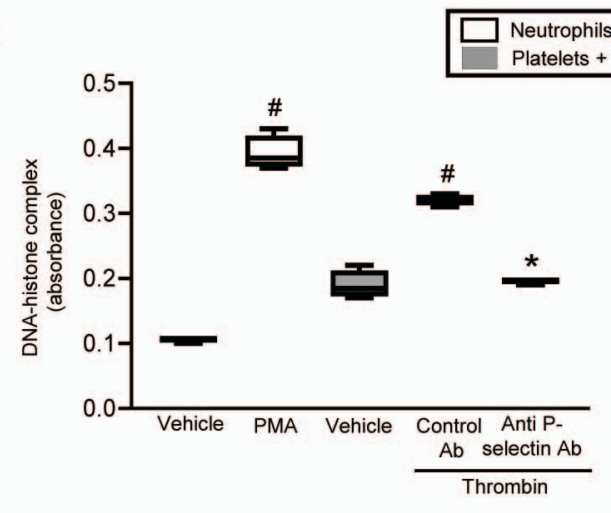

Figure 2. Platelet-neutrophil interactions in AP. A) Platelet-neutrophil aggregates were quantified as the percentage of neutrophils (Ly6G ${ }^{+}$) binding platelets $\left(\mathrm{CD} 41^{+}\right)$in the blood as described in Materials and Methods. B) Aggregate data on platelet-neutrophil aggregates. C) Plasma levels of DNA-histone complexes. Pancreatic levels of D) histone 3 and E) histone 4. F) Scanning electron microscopy exhibiting extracellular web-like structures in the inflamed pancreas. Scale bar $=25 \mu \mathrm{m} . \mathrm{G}$ ) NETs are denoted in pink color. H) Transmission electron microscopy of the indicated area of interest from Figure 1F incubated with gold-labeled anti-histone 4 (large gold particles) and anti-elastase (small gold particles) antibodies. Scale bar $=0.25 \mu \mathrm{m}$. I) DNA-histone complex formation. Mixtures of neutrophils and platelets were stimulated with thrombin and treated with a control or an anti-P-selectin antibody (Ab). PMA-stimulated neutrophils served as a positive control. Pancreatitis (grey boxes) was induced by infusion of sodium taurocholate $(5 \%)$ into the pancreatic duct. Sham mice (white boxes) were infused with saline alone. Animals were treated with i.v. injections of a control or an anti-P-selectin antibody (Ab) as described in Materials and Methods. 


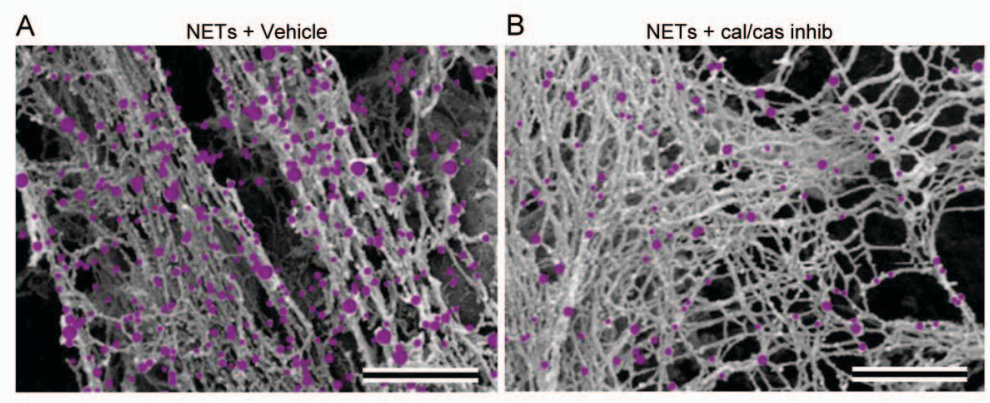

D

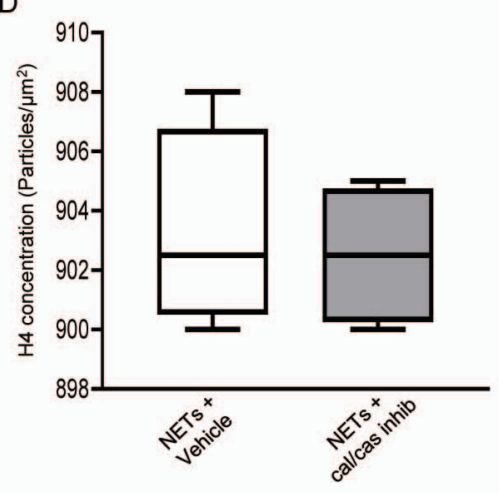

G

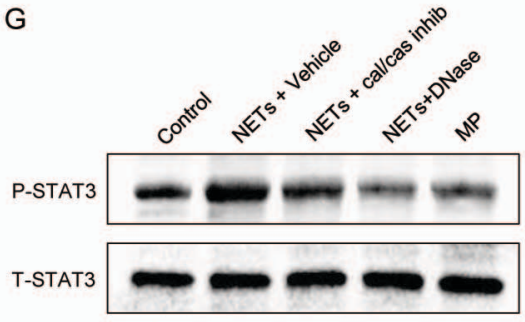

E

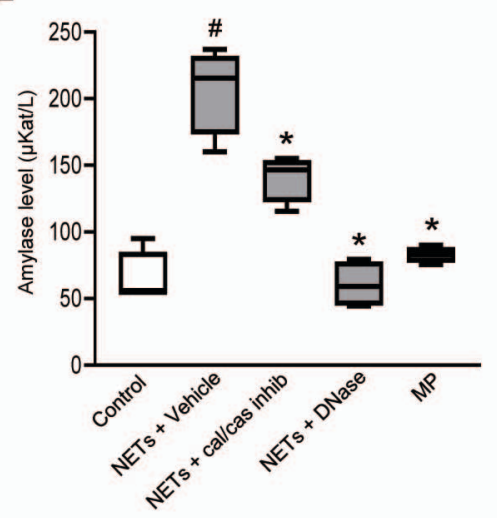

$\mathrm{H}$

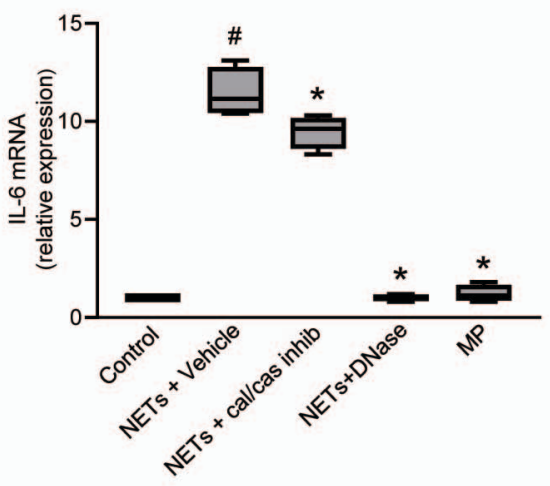

$\mathrm{C}$

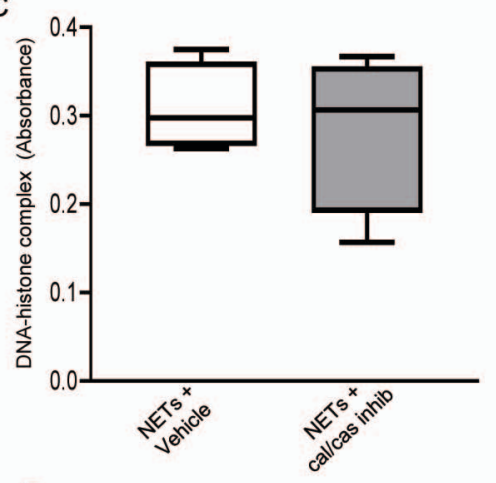

F

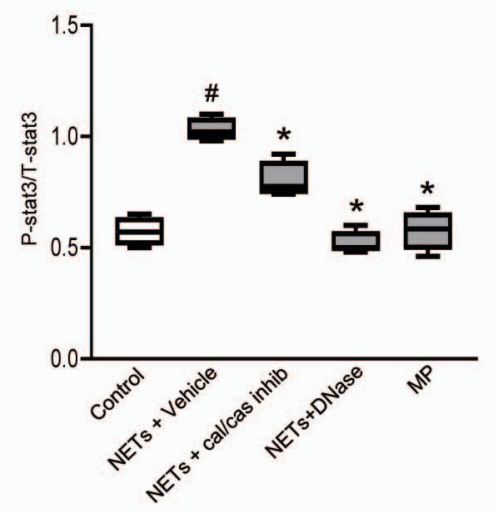

I

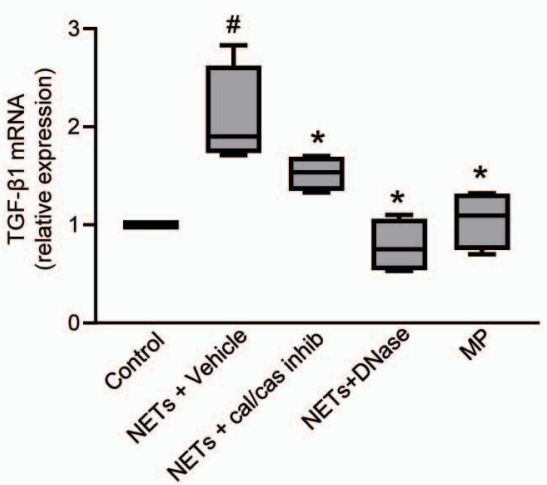

Figure 3. Functional role of NET-MP complexes. Scanning electron microscopy showing NETs generated from PMA-stimulated bone marrow neutrophils in the presence of A) vehicle or B) caspase (50 $\mu \mathrm{M}, \mathrm{Z}-\mathrm{VAD}-\mathrm{FMK})$ and calpain ( $25 \mu \mathrm{M}, \mathrm{PD} 150606)$ inhibitors. MPs are denoted in pink color. Scale bar $=1 \mu \mathrm{m}$. C) DNA-histone complex formation and D) histone 4 concentration on NETs with and without MPs. E) Amylase secretion, F) aggregate data showing phosphorylated STAT-3 normalized to total STAT-3 and G) a representative western blot of phosphorylated STAT-3 and total STAT-3 as well as gene expression of H) IL-6 and I) TGFB1. Acinar cells were stimulated with NETs (grey boxes) formed in the presence of vehicle, a combination of caspase and calpain inhibitors or DNase as well as with isolated neutrophil-derived MPs. Data represent median (25-75 percentile); whiskers extend from the minimum to the maximum values and $n=4 .{ }^{\sharp} P<0.05 v s$. Control and $* P<0.05 v s$. Vehicle+NETs (Mann-Whitney test). 

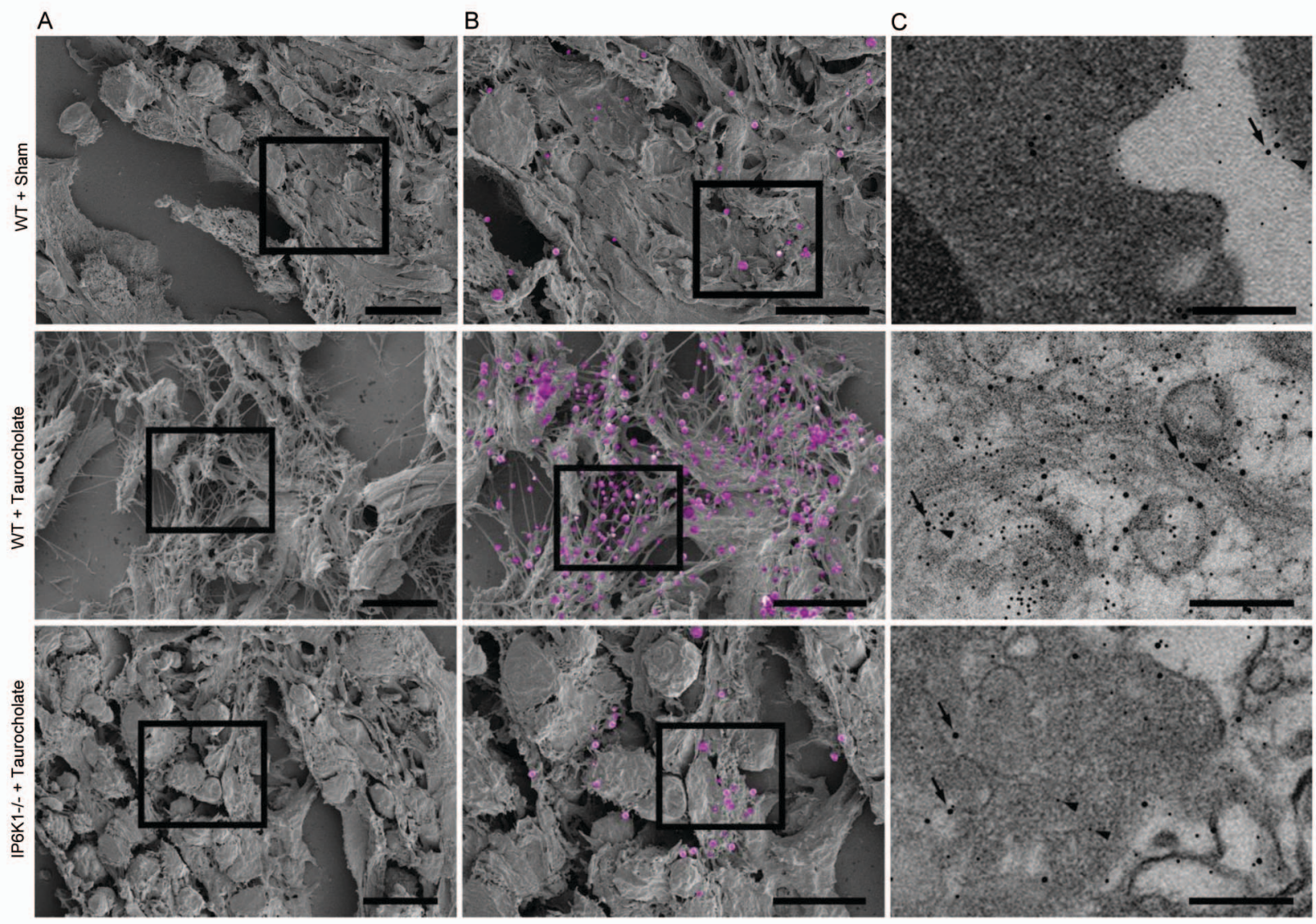

D

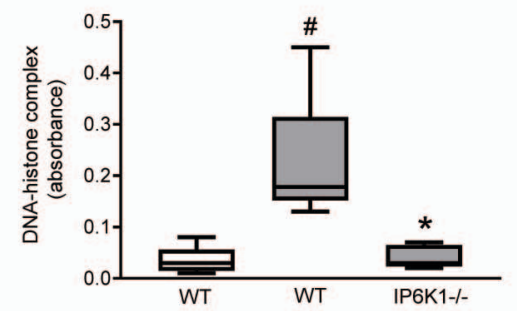

E

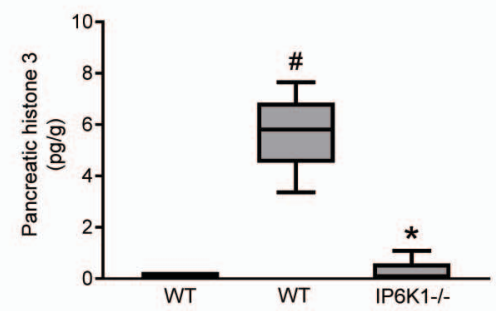

F

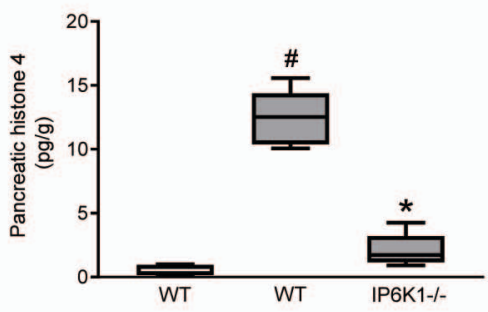

Figure 4. IP6K1-dependent formation of NET-MP complexes in AP. A) Scanning electron microscopy exhibiting extracellular weblike structures in the inflamed pancreas. Scale bar $=5 \mu \mathrm{m}$. B) A higher magnification of the indicated area of interest from Figure $1 \mathrm{~A}$ showing fibrillar MPs attached to NETs in the inflamed pancreas. MPs are denoted in pink color. Scale bar $=2 \mu \mathrm{m}$. C) Transmission electron microscopy of the indicated area of interest from Figure 1B incubated with gold-labeled anti-Mac-1 (large gold particles) and anti-CD41 (small gold particles) antibodies. Scale bar $=0.25 \mu \mathrm{m}$. D) Plasma levels of DNA-histone complexes. Pancreatic levels of E) histone 3 and F) histone 4. Pancreatitis (grey boxes) was induced by infusion of sodium taurocholate (5\%) into the pancreatic duct of wild-type (WT) and IP6K1 gene-deficient (IP6K1-/-) mice. Sham mice (white boxes) were infused with saline alone. Samples were collected 24 hours after induction of pancreatitis. Data represent median (25-75 percentile); whiskers extend from the minimum to the maximum values and $n=5$. ${ }^{\#} P<0.05$ versus Sham WT mice and $* P<0.05$ versus taurochoalte+IP6K1-/- mice (Mann-Whitney test). 


\section{Figure 5}
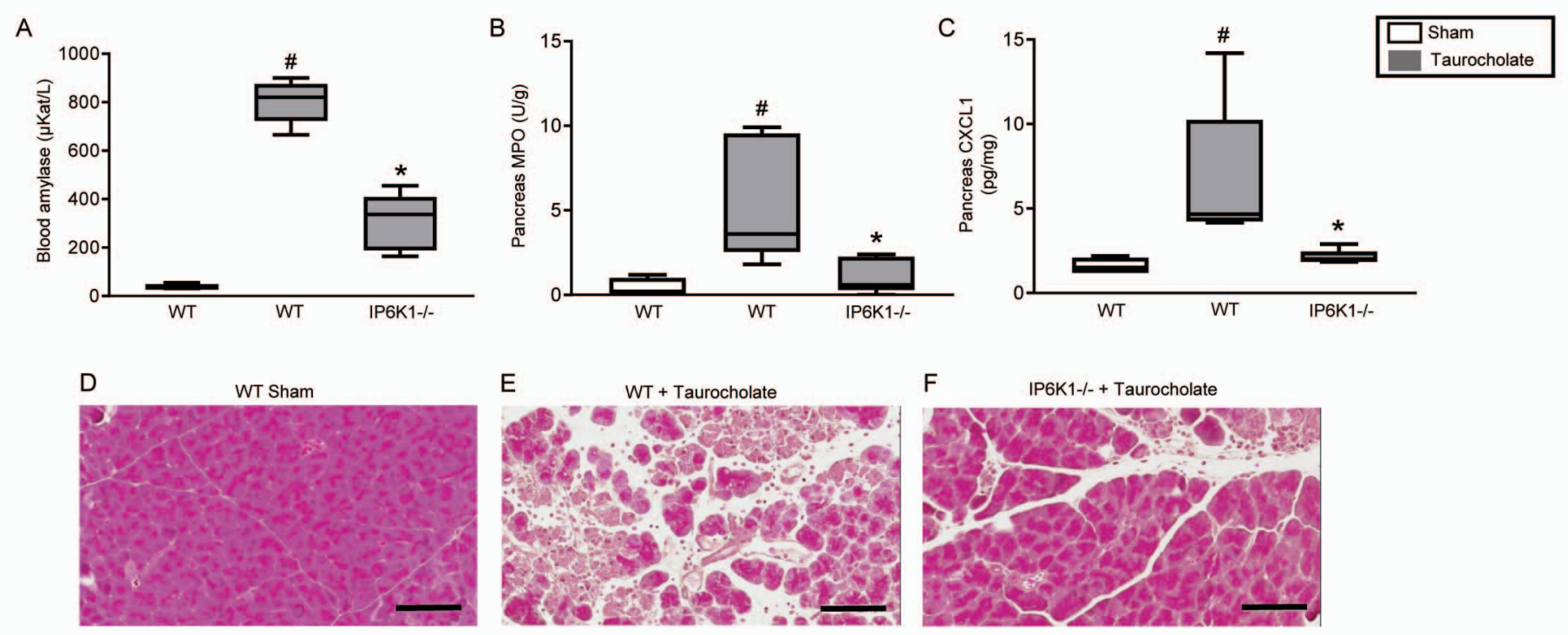

G

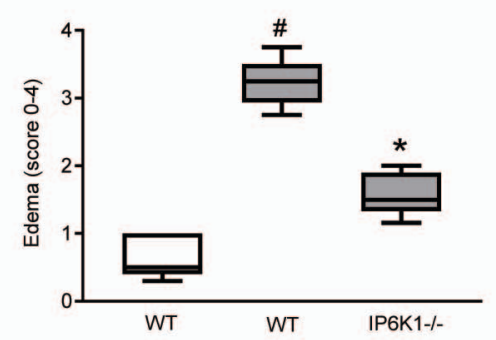

$\mathrm{K}$

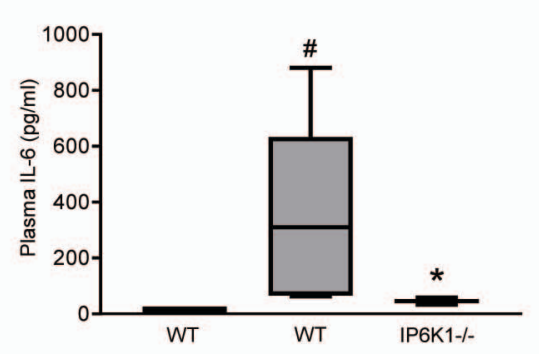

L
$\mathrm{H}$

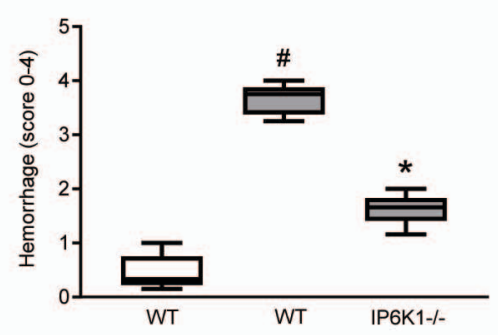

I

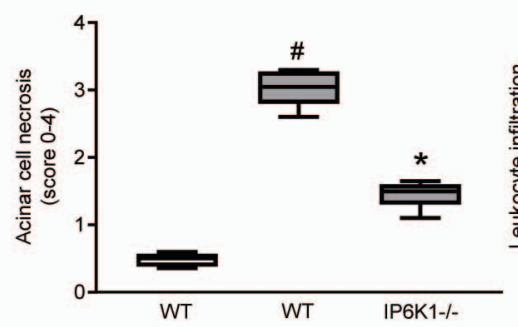

$\mathrm{J}$
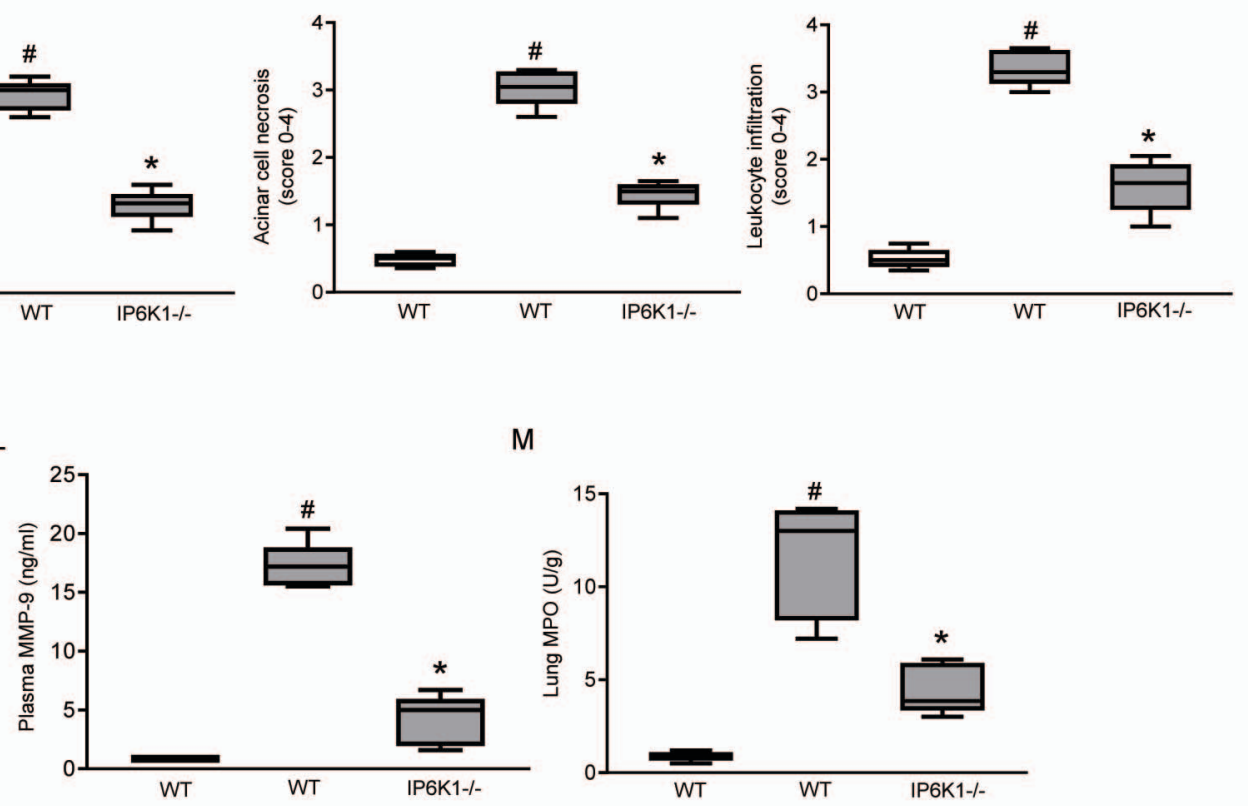

M

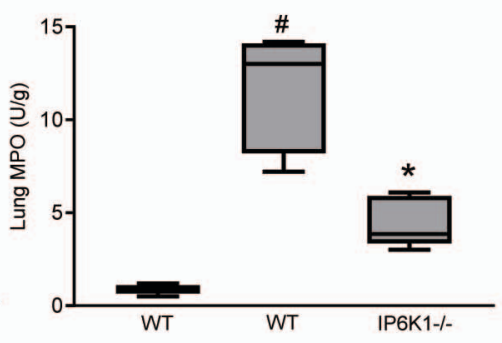

Figure 5. IP6K1-dependent inflammation and tissue damage in AP. Quantitative measurements of A) blood amylase levels, pancreatic B) MPO and C) CXCL1 levels. D-F) Representative hematoxylin \& eosin sections of the head of the pancreas from indicated groups. Scale bar $=100 \mu \mathrm{m}$. Histological quantification of $\mathrm{G}$ ) edema, H) hemorrhage, $\mathrm{I}$ ) acinar cell necrosis and J) leukocyte infiltration. Plasma levels of $\mathrm{K}$ ) IL-6 and L) MMP-9 as well as M) lung levels of MPO. Pancreatitis (grey boxes) was induced by infusion of sodium taurocholate (5\%) into the pancreatic duct of wild-type (WT) and IP6K1 gene-deficient (IP6K1 -/-) mice. Sham mice (white boxes) were infused with saline alone. Samples were collected 24 hours after induction of pancreatitis. Data represent median ( $25-75$ percentile); whiskers extend from the minimum to the maximum values and $\mathrm{n}=5$. ${ }^{\#} P<0.05$ versus Sham WT mice and $* P<0.05$ versus taurochoalte+IP6K1-/- mice (MannWhitney test). 
Figure 6
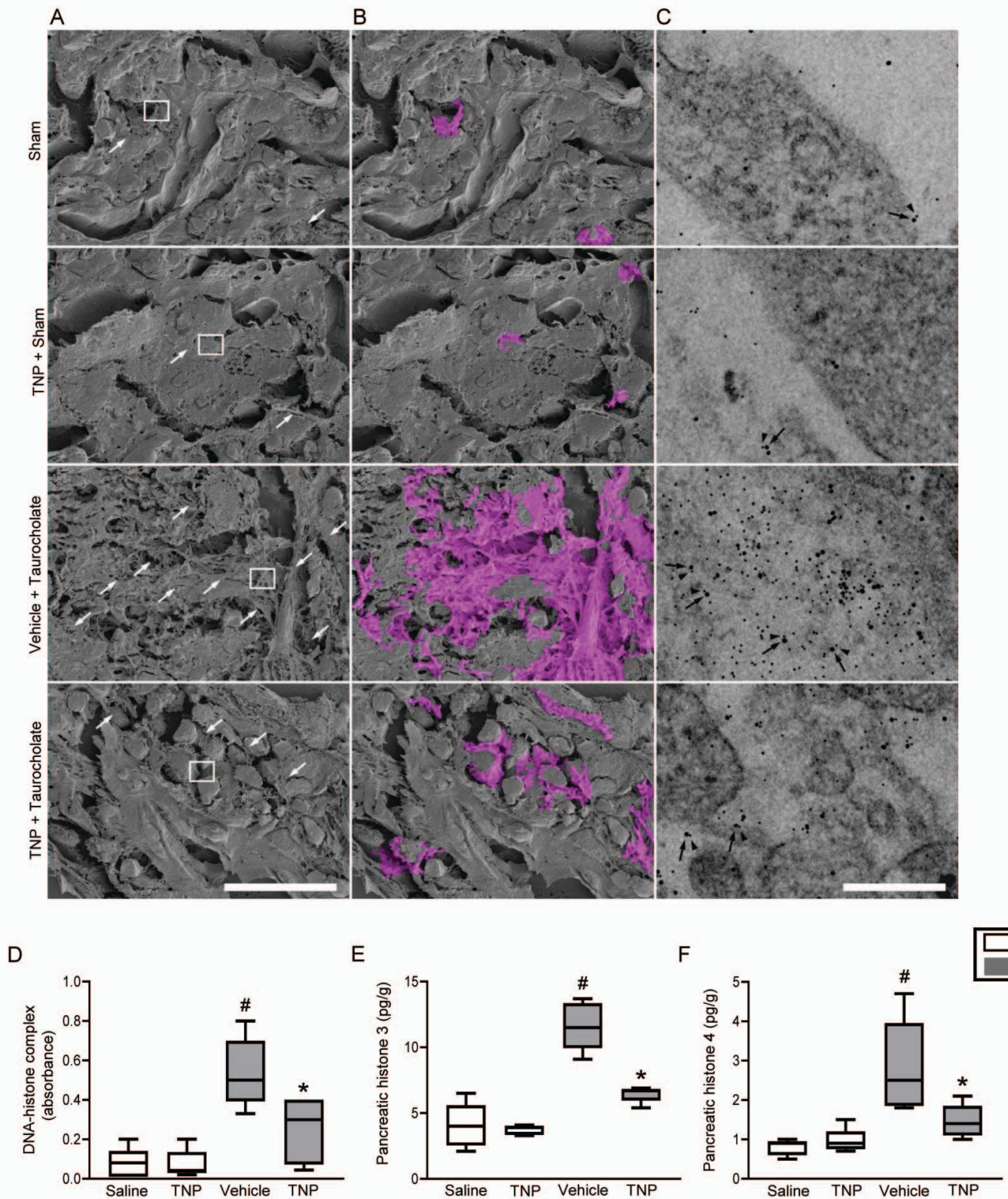

Figure 6. TNP inhibits NETs formation in AP. A) Scanning electron microscopy exhibiting extracellular web-like structures in the inflamed pancreas. Scale bar $=25 \mu \mathrm{m}$. B) NETs denoted in pink color. C) Transmission electron microscopy of the indicated area of interest from Figure 1A incubated with gold-labeled anti-histone 4 (large gold particles, arrows) and antielastase (small gold particles, arrowheads) antibodies. Scale bar $=0.25 \mu \mathrm{m}$. D) Plasma levels of DNA-histone complexes. Pancreatic levels of E) histone 3 and F) histone 4. Pancreatitis (grey boxes) was induced by infusion of sodium taurocholate (5\%) into the pancreatic duct. Sham mice (white boxes) were infused with saline alone. Animals were treated with i.p. injections of the vehicle (PBS) or TNP as described in Materials and Methods. Samples were collected 24 hours after induction of pancreatitis. Data represent median (25-75 percentile); whiskers extend from the minimum to the maximum values and $n=$ 5. ${ }^{\#} P<0.05$ versus $\mathrm{PBS}+$ Sham and $* P<0.05$ versus Vehicle+taurocholate (Mann-Whitney test). 
A

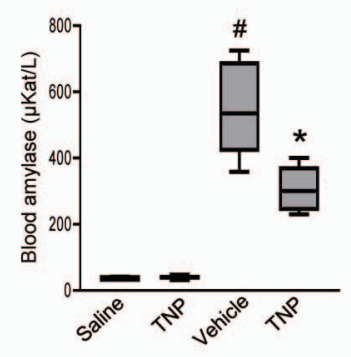

$E$
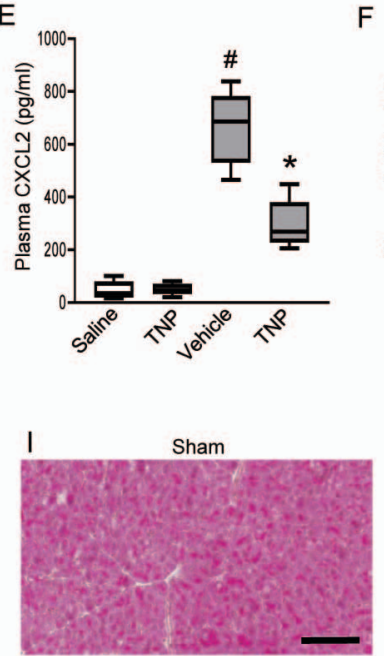

B

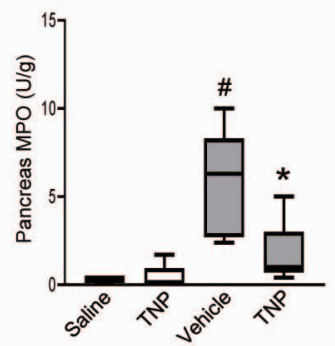

F

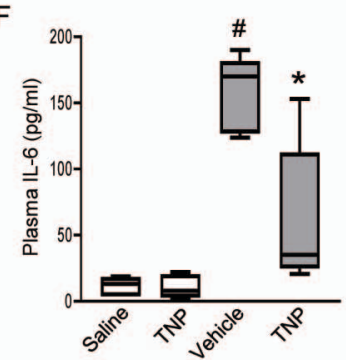

C

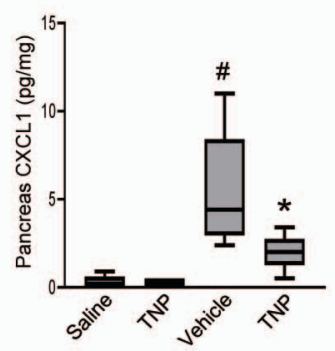

G

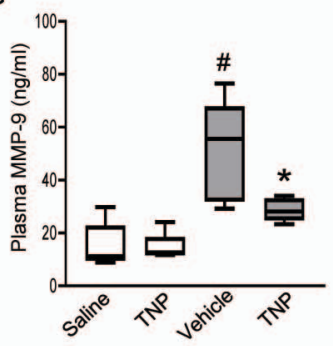

D

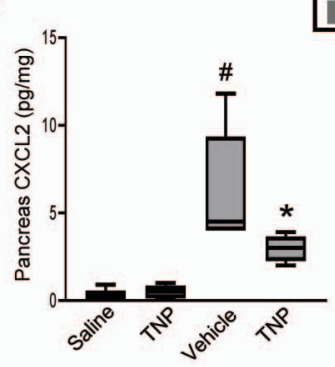

$\mathrm{H}$

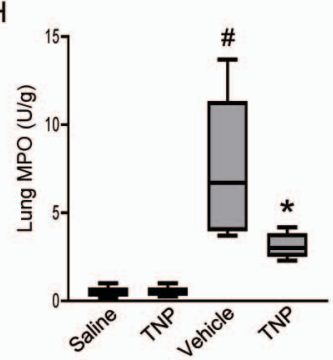

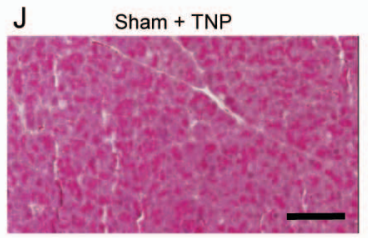
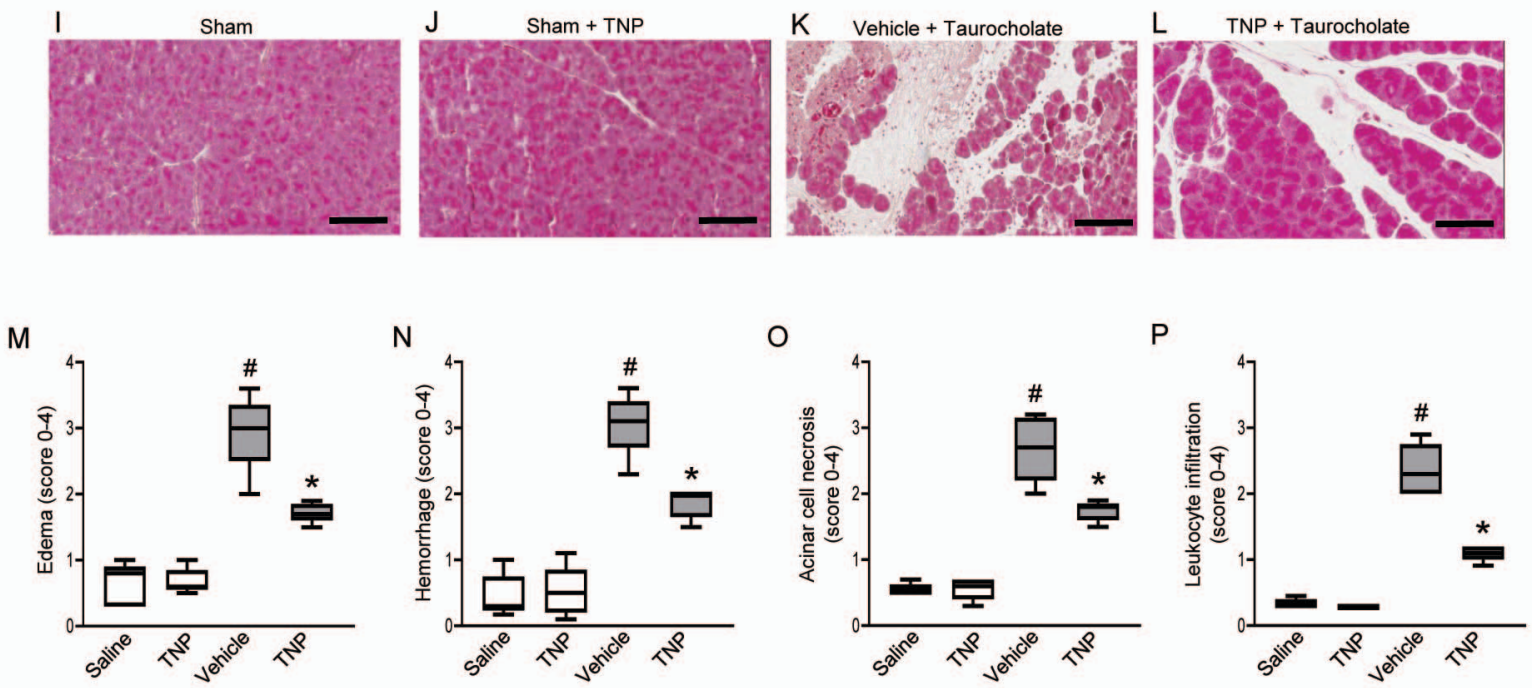

Figure 7. TNP inhibits inflammation and tissue damage in AP. Quantitative measurements of A) blood amylase levels, pancreatic B) MPO, C) CXCL1 and D) CXCL2 levels. Plasma levels of E) CXCL2, F) IL-6 and G) MMP-9 as well as H) lung levels of MPO. I-L) Representative hematoxylin \& eosin sections of the head of the pancreas from indicated groups. Scale bar $=100 \mu \mathrm{m}$. Histological quantification of $\mathrm{M}$ ) edema, $\mathrm{N}$ ) hemorrhage, $\mathrm{O}$ ) acinar cell necrosis and $\mathrm{P}$ ) leukocyte infiltration. Pancreatitis (grey boxes) was induced by infusion of sodium taurocholate $(5 \%)$ into the pancreatic duct. Sham mice (white boxes) were infused with saline alone. Animals were treated with i.p. injections of the vehicle or TNP as described in Materials and Methods. Samples were collected 24 hours after induction of pancreatitis. Data represent median (25-75 percentile); whiskers extend from the minimum to the maximum values and $\mathrm{n}=5$. ${ }^{\#} P<0.05$ versus sham mice and $* P<0.05$ versus Vehicle+taurocholate (Mann-Whitney test). 
Figure 8

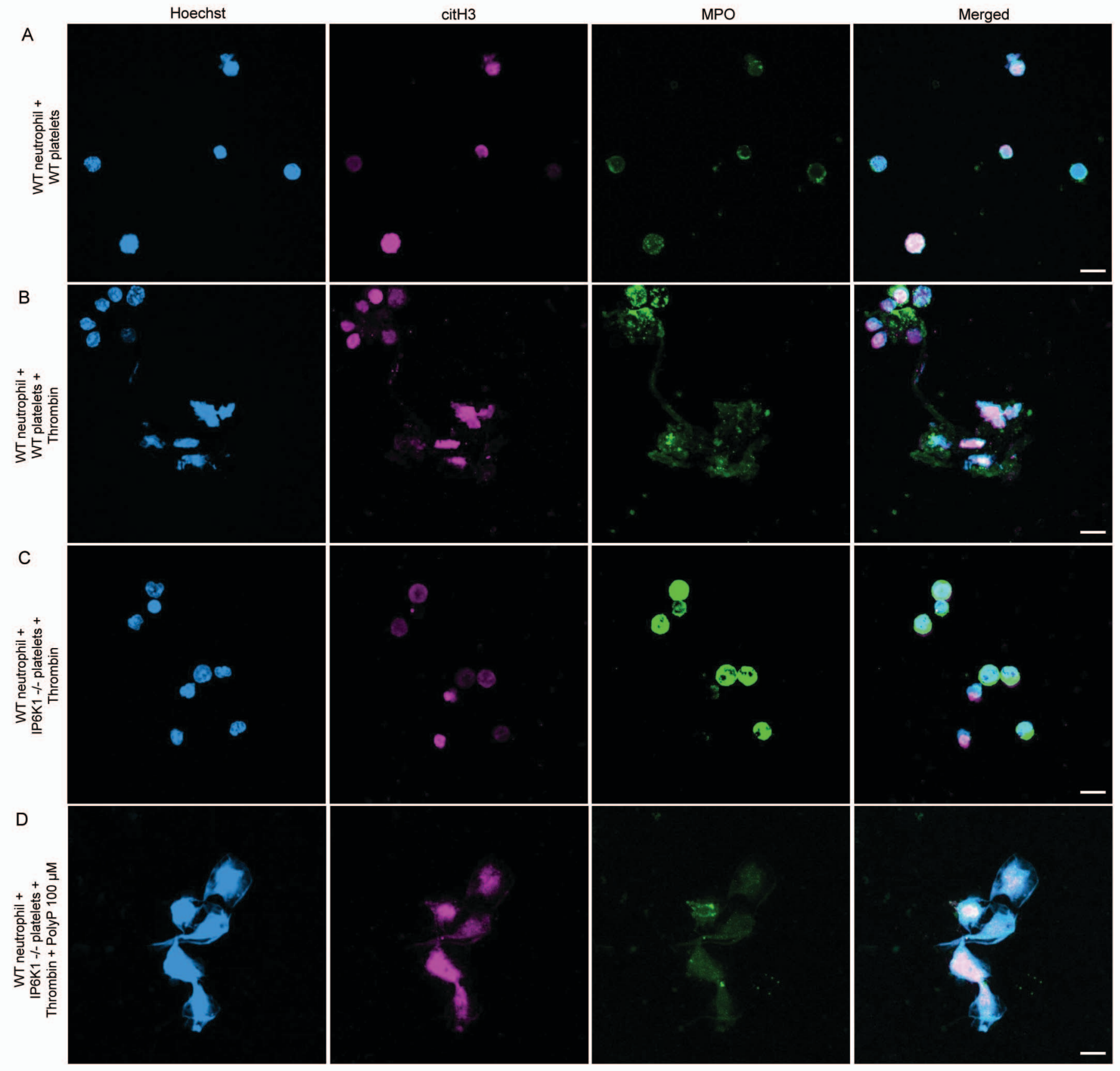

Figure 8. PolyP-mediated formation of NETs. NETs were generated from isolated neutrophils by incubating with isolated wild-type or IP6K1-/- platelets with or without thrombin $(0.2 \mathrm{U} / \mathrm{ml})$ and with or without PolyP $(100 \mu \mathrm{M})$ over coverslips at $37^{\circ} \mathrm{C}$ for 3 hours. Neutrophils were fixed and permeabilized and then immune-stained with antibodies to citrullinated histone 3 (citH3), myeloperoxidase (MPO), and DNA was counterstained with Hoechst 33342. One representative experiment of four independent experiments. Scale bars $=10 \mu \mathrm{m}$. 